\title{
Top-Down Fabricated Silicon Nanowire Arrays for Field-Effect Detection of Prostate-Specific Antigen
}

\author{
Dipti Rani, ${ }^{\dagger, \perp}$ Vivek Pachauri, ${ }^{\dagger, \# \odot ~ N a r a y a n a n ~ M a d a b o o s i, ~}{ }^{\ddagger, \nabla}$ Pawan Jolly, ${ }^{\S, \bigcirc}$ Xuan-Thang Vu, ${ }^{\dagger, \|, \#}$ \\ Pedro Estrela, ${ }^{\S}$ (1) Virginia Chu, ${ }^{*}$ João Pedro Conde, ${ }^{*}$ and Sven Ingebrandt*, ${ }^{\dagger, \#(1)}$
}

${ }^{\dagger}$ Department of Informatics and Microsystem Technology, University of Applied Sciences Kaiserslautern, Amerikastrasse 1, 66482 Zweibrücken, Germany

${ }^{\ddagger}$ INESC Microsistemas e Nanotecnologias, Rua Alves Redol, 91000-029 Lisbon, Portugal

${ }^{\S}$ Department of Electronic and Electrical Engineering, University of Bath, BA2 7AY Bath, United Kingdom

"Institute of Physics I, RWTH Aachen University, Sommerfeldstr. 14, 52074 Aachen, Germany

Supporting Information

\begin{abstract}
Highly sensitive electrical detection of biomarkers for the early stage screening of cancer is desired for future, ultrafast diagnostic platforms. In the case of prostate cancer ( $\mathrm{PCa})$, the prostate-specific antigen (PSA) is of prime interest and its detection in combination with other PCa-relevant biomarkers in a multiplex approach is advised. Toward this goal, we demonstrate the label-free, potentiometric detection of PSA with silicon nanowire ion-sensitive field-effect transistor ( $\mathrm{Si}$ NW-ISFET) arrays. To realize the field-effect detection, we utilized the DNA aptamerreceptors specific for PSA, which were covalently and site-specifically immobilized on Si NW-ISFETs. The platform was used for quantitative detection of PSA and the change in threshold voltage of the Si NW-ISEFTs was correlated with the concentration of PSA. Concentration-dependent measurements were done in a wide range of $1 \mathrm{pg} / \mathrm{mL}$ to $1 \mu \mathrm{g} / \mathrm{mL}$, which covers the clinical range of interest. To confirm the PSA-DNA aptamer

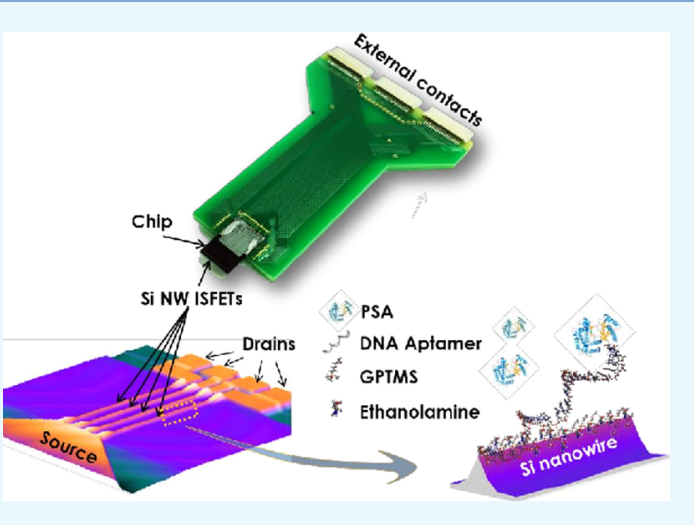
binding on the Si NW surfaces, a sandwich-immunoassay based on chemiluminescence was implemented. The electrical approach using the Si NW-ISFET platform shows a lower limit of detection and a wide dynamic range of the assay. In future, our platform should be utilized to detect multiple biomarkers in one assay to obtain more reliable information about cancerrelated diseases.
\end{abstract}

\section{INTRODUCTION}

Nanoscale field-effect transistors (FETs) have emerged as versatile and multiphasic technological tools for the realization of high-performance sensor applications. ${ }^{1}$ Chemical sensing or biosensing in solutions require the operation of the FETs in an electrochemical-gate configuration. This is the classical ionsensitive field-effect transistor (ISFET) concept, where the FET response is determined by changes in the surface potential at the solid-liquid interface and by the composition of the electrical double layer (EDL), which are both influenced by the binding of charged molecules. ${ }^{2,3}$ The usage of onedimensional semiconductors such as silicon nanowires ( $\mathrm{Si}$ NWs) for ISFET platforms is advantageous because of their high sensitivities toward surface-potential changes. Therefore, Si NW-ISFETs are utilized for high-performance, "label-free" electrical biosensors. ${ }^{4-6}$ In recent years, Si NW-ISFETs have been described as an ideal candidate for different bioassays with an ever-increasing focus on novel concepts dealing with label-free, continuous screening of various biomolecules from complex biological media toward point-of-care (PoC) applications. $^{7-10}$
However, the use of nanoscale ISFETs for real applications remains challenging due to intrinsic limitations. Their electrical characteristics are largely influenced by the composition of the EDL. Thereby, they suffer from diminished performance for biodetection in physiological solutions due to EDL contraction or Debye screening of the biomolecule charges (typically $<1$ $\mathrm{nm}$ for physiological saline or biological fluids). ${ }^{8,11,12}$ Strategies to overcome this Debye screening for PoC biosensor applications in blood serum have included physical manipulation of the solid-liquid interface by polymer spacer layers, rendering of nanoscale features, or alternating the working requirements. ${ }^{13-17}$ Alternative approaches to counteract Debye screening rely on the use of nanoscale capacitors operated at high frequencies and the impedimetric readout strategies with ISFETs. ${ }^{18-20}$ Here, microfluidics-integrated sensors have demonstrated great potential toward label-free

Received: May 14, 2018

Accepted: July 18, 2018

Published: August 1, 2018 

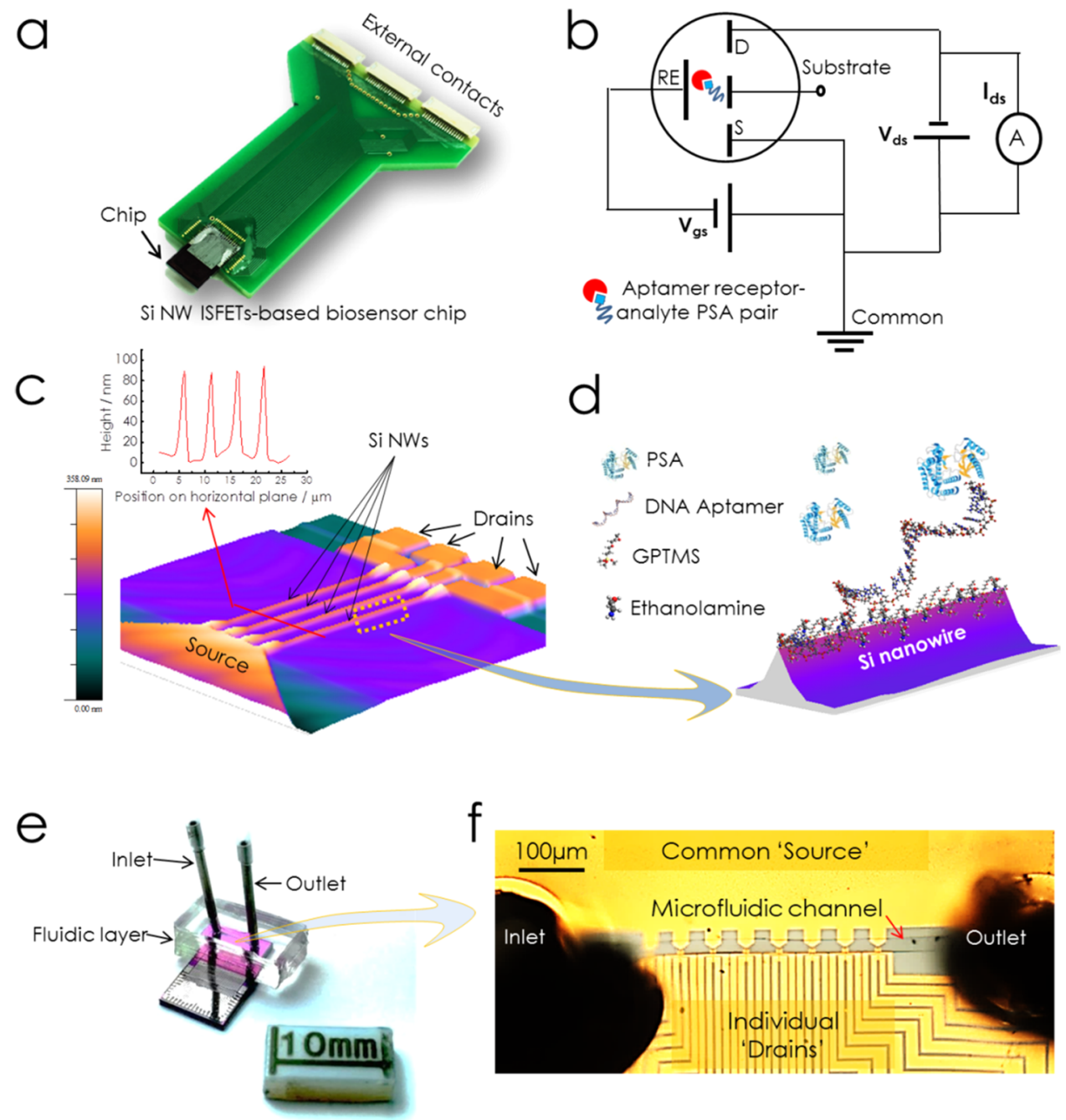

Figure 1. Realization of the Si NW-ISFET sensor platform: photograph of a top-down fabricated sensor chip with Si NW-ISFETs mounted onto a PCB and wire-bonded to facilitate external electrical contacts (a), an electrical circuit diagram for the operation of the Si NW sensors (b), surface characterization of a set of four Si NWs using AFM and representation as a three-dimensional image showing identical structural characteristics (c), schematic illustration of the surface functionalization and build-up of the biofunctional layer on the Si NW surface for label-free electrical detection of PSA (d), photograph of a sensor chip with a fluidic layer assembled on top to facilitate easy handling of analytes (e), and microscopic top view of the microfluidic channel in poly(dimethylsiloxane) (PDMS) aligned over 8 sets of Si NWs on top of the 32-channel Si NW-ISFET chip, also showing common source and individual drain contact lines (f).

bioassays for screening of a variety of biomarkers directly in physiological saline or human serum. ${ }^{10,21-24}$

$\mathrm{Si}$ NW-ISFET concepts are especially interesting for industrial upscale due to the possible mass-production using state-of-the-art, top-down nanofabrication methods (e.g., nanoimprint lithography (NIL) or extreme ultraviolet lithography). ${ }^{25,26}$ In our previous work, we described a NIL approach for wafer-scale, high-density fabrication of Si NWISFET arrays with identical sensor characteristics. ${ }^{27}$ In this work, we deploy these Si NW-ISFETs as label-free electrical biosensors to realize simple and direct potentiometric screening of prostate-specific antigen (PSA), a $33 \mathrm{kDa}$ singlechain glycoprotein. A healthy man has a PSA level $<4 \mathrm{ng} / \mathrm{mL}$ in serum, whereas increased PSA concentrations may indicate a risk of prostate cancer. Also, the increased rate of PSA level over time is a solid diagnostic evidence and therefore multiple and swift tests over time would enable better healthcare. For risk stratification, an increased serum PSA level is usually complemented with biopsies followed by tissue examination. ${ }^{28}$ These procedures are sometimes unnecessary and can even cause over diagnosis of risks in some patients. ${ }^{29-34}$ The direct screening and close monitoring of PSA together with newer biomarkers such as glycoproteins and micro-RNAs may reduce the painful tissue examination procedures in the future.

Unlike other sensor platforms described in literature for the label-free detection of PSA with PSA-specific antibodies as receptors, our Si NW-ISFETs were functionalized with DNA aptamers specific to PSA. ${ }^{35-44}$ Specially designed aptamers mimicking the binding site conformations of target analytes have a strong advantage over antibody receptors, particularly for field-effect sensing due to their much smaller size. ${ }^{13,42,45-48}$ In this work, we show a high specificity and a robust binding of PSA toward the aptamer functionalized Si NW surfaces. The small sizes typically within a few nanometers and the negatively charged DNA sequences of the aptamers render the solidliquid interface with a defined surface charge density. Therefore, the use of aptamers as biofunctionalization layer is advantageous for field-effect sensing of large biomolecules. This is demonstrated in this work for screening of PSA with a dynamic concentration range spanning over 5 orders of 

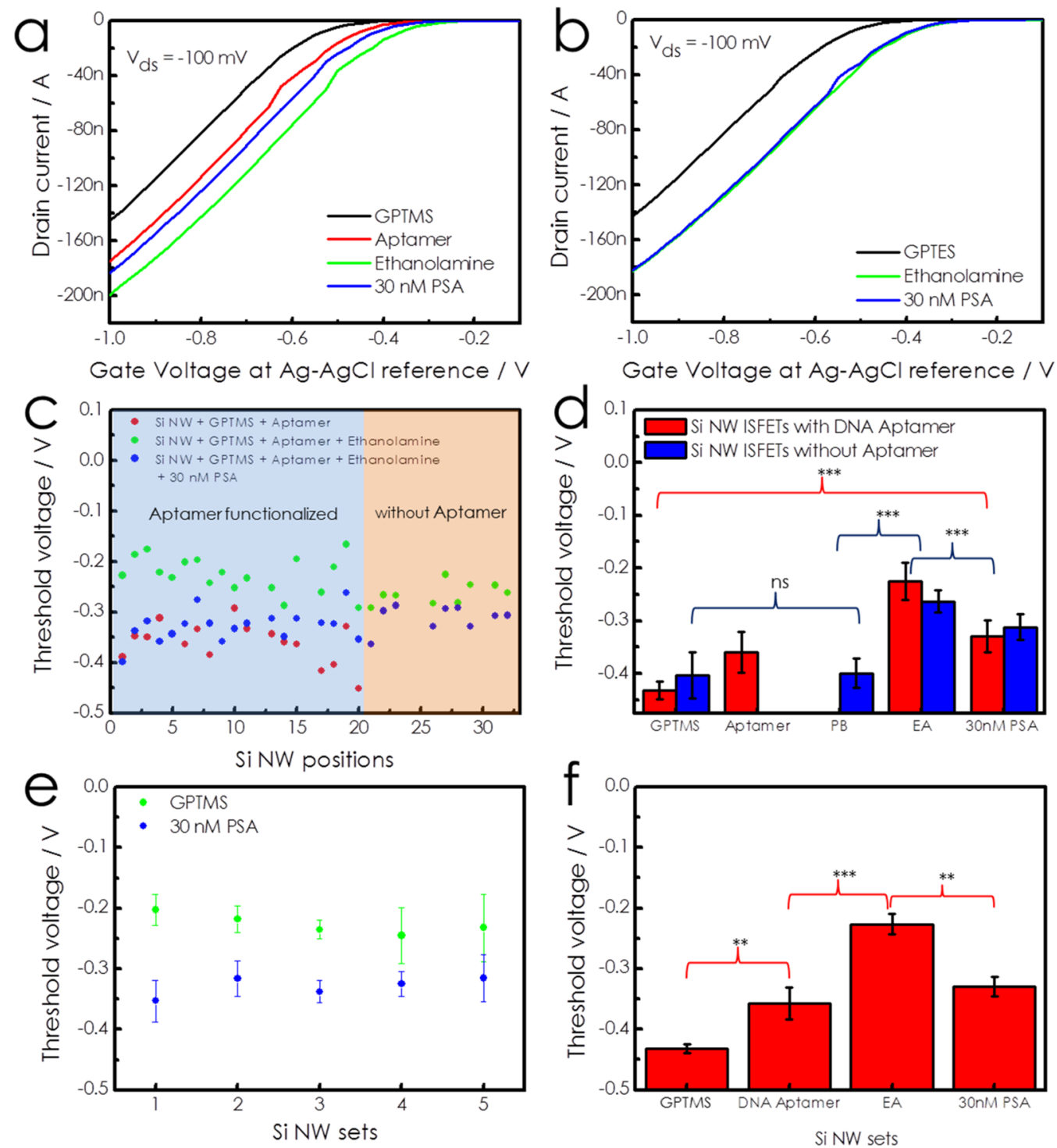

Figure 2. Statistical evaluation of the sensing behavior of the Si NW-ISFETs for the detection of PSA: shift in $V_{\text {th }}$ of a typical Si NW-ISFET after different surface immobilization steps including GPTMS, aptamer, EA functionalization, and $30 \mathrm{nM}$ PSA binding (a), transfer characteristics of a Si NW-ISFET without aptamer functionalization as a negative control (b), sensor responses of 27 out of $32 \mathrm{Si}$ NWs from a single sensor chip with and without aptamer functionalization. The recordings are shown in one graph as the change in $V_{\text {th }}$ values before and after binding of $30 \mathrm{nM}$ PSA (c). The large number of channels on one chip enables us to statistically evaluate multiple experiments. The histogram shows the average changes in $V_{\text {th }}$ during surface immobilization steps with and without aptamers. A statistical analysis of these changes was done with a paired $t$-test (d). The dot chart shows the change in average $V_{\text {th }}$ of different Si NW sets (four Si NWs each) on one-sensor chip before and after analyte binding (e). Average $V_{\text {th }}$ change of these Si NW sets at each immobilization step is represented as average value \pm standard deviation. When evaluating all sensors, the paired $t$-test shows significance for all steps $(* * *=P<0.001, * *=P<0.01$ and $\mathrm{ns}$ for nonsignificant) (f).

magnitude while fully covering the clinically relevant concentration range.

Furthermore, we compare the response of the aptamermodified Si NW-ISFETs with microscale Si ISFETs. ${ }^{49}$ The nanoscale devices show a much better response for label-free electrical biosensing. As a control assay, we detected the binding of PSA by chemiluminescence on the chip surfaces, which also confirmed the highly-specific binding to the aptamer receptors. The $\mathrm{Si}$ NW-ISFET aptasensor arrays achieved a high performance for the label-free detection of PSA biomarkers in a concentration range from $3 \mathrm{pM}$ to $30 \mathrm{nM}$ (10 $\mathrm{pg} / \mathrm{mL}$ to $1 \mu \mathrm{g} / \mathrm{mL}$ ). Our optimized Si NW-ISFET aptasensor platform therefore offers a straightforward fieldeffect detection of globular biomolecules. Enabled with a miniaturized handheld readout, multiplexing approaches combining similar sensor arrays for other candidate biomarkers on this sensor platform can be promising in the near future for high-throughput clinical assays towards PoC screening of cancer biomarkers in general. ${ }^{21,50-53}$

\section{RESULTS AND DISCUSSION}

Top-down nanofabrication of enhancement-mode, p-type Si NW-ISFETs with an optimized lithography protocol was earlier developed in our group and the realization of highaspect-ratio Si NWs on 4 in. silicon-on-insulator (SOI) wafers was demonstrated. ${ }^{27}$ It was confirmed that the resulting arrays of $\mathrm{Si}$ NWs exhibited high-quality $\mathrm{SiO}_{2}$ gate dielectrics after anisotropic wet etching and dry oxidation, resulting in superior 
sensor characteristics with low electrical hysteresis and identical sensors over wafer scale. The usage of p-type devices has a chemical reason in particular when only $\mathrm{SiO}_{2}$ is used as gate dielectrics. In case of n-type, the cations from the electrolyte solution intercalate into the porous oxide driven by the gate bias voltage causing break down of the dielectrics and malfunctioning of the sensors. Better performance is reported for other gate dielectrics like $\mathrm{Al}_{2} \mathrm{O}_{3}$ or $\mathrm{HfO}_{2}$, which we aim to integrate in a later stage of our project as well. Fabrication details of the devices are summarized in the Materials and Methods section. A typical sensor chip with $\mathrm{Si}$ NWs arrays is shown in Figure 1. Figure 1a shows a photograph of a sensor chip that was mounted on a specially designed printed circuit board (PCB) to enable external electrical contacts for the Si NW-ISFET source and drains. Figure $1 b$ illustrates an electrical circuit diagram for the operation of the Si NW-ISFETs. As it can be seen from Figure $1 b$, the source is grounded, whereas a negative bias is applied to the drain, resulting in a drainsource voltage $V_{\mathrm{ds}}$. An $\mathrm{Ag}-\mathrm{AgCl}$ reference electrode is immersed into the electrolyte solution and used to apply the front-gate voltage $V_{\mathrm{gs}}$, whereas the drain-source current $I_{\mathrm{ds}}$ is measured via an amplifier. In this configuration, the back-gate contact was grounded and therefore fixed to the same potential as the source contact. Figure $1 c$ shows a three-dimensional representation of an atomic force microscopy (AFM) scan of a typical set of Si NWs connected to a common source and four individual drains. Each $\mathrm{Si} \mathrm{NW}$ with a trapezoidal cross section measured $14 \mu \mathrm{m}$ in length, $67 \mathrm{~nm}$ in height, and $250 \mathrm{~nm}$ in width at the base. The Si NWs had a thermally grown gate oxide layer $(6-8 \mathrm{~nm})$ for ISFET operation as well as for providing hydroxyl groups for subsequent chemical surface modifications.

The schematic illustration in Figure 1d shows the covalently functionalized aptamer sequences ( 32 nucleotides) as a PSAspecific biofunctional layer on a Si NW. More information on the aptamer sequence and the surface functionalization protocols is provided in the Materials and Methods section as well as in the Supporting Information (Figure S1). Figure le,f shows the integration of a microfluidic channel on top of the sensor chip realized in a poly(dimethylsiloxane) (PDMS) layer. The microfluidic channel was aligned over the Si NW sets, which can be seen in the microscopic image taken through the PDMS layer (Figure 1f).

Aptamer-functionalized Si NW-ISFETs were then deployed for the electrical detection of PSA, and initial findings on the sensor performance are summarized in Figure 2. Measurements were carried out in phosphate buffer (PB) at $\mathrm{pH} 7.4$ while applying a drain-source bias of $-100 \mathrm{mV}$ and sweeping a gate voltage against an $\mathrm{Ag} / \mathrm{AgCl}$ reference electrode from -1 $\mathrm{V}$ to $-100 \mathrm{mV}$. Transfer characteristics of a typical Si NWISFET recorded with these parameters are shown in Figure 2a. The black curve in Figure 2a represents the transfer characteristics of a Si NW-ISFET modified with 3-glycidoxypropyltrimethoxysiloxane (GPTMS) using a high-quality gasphase silanization protocol. ${ }^{54}$ In the field of biosensor research also, other abbreviations for this type of silane are used such as GPTES or GOPS. Main advantage of using this silane instead of the more common (3-aminopropyl)triethoxysilane is that cross-linking with additional molecules such as succinic anhydride can be omitted. ${ }^{55}$ The direct link of aminofunctionalized capture molecules to the GPTMS layer is advantageous for the field-effect detection resulting in a smaller distance of the analyte molecules to the sensor surface after binding. A threshold voltage $\left(V_{\text {th }}\right)$ shift toward positive gate voltages was observed after surface functionalization with PSAspecific aptamers, shown as the red curve in Figure 2a. Aminomodified aptamers were used as receptors. They can covalently bind to the glycidol functional groups of GPTMS while introducing additional negative charges to the solid-liquid interface. Therefore, a decrease in $V_{\text {th }}$ of the Si NWs upon binding of aptamers is observed. ${ }^{53,56}$ The successful functionalization of aptamers onto the Si NW surfaces was also confirmed by AFM measurements, which are discussed in the Supporting Information in Figure S2. The average size of the PSA-specific aptamers with 32 nucleotides is about $10 \mathrm{~nm}$ in length and about $1.2-1.8 \mathrm{~nm}$ in thickness. In a $10 \mathrm{mM}$ ionic strength $\mathrm{PB}$, the Debye length is about $2 \mathrm{~nm}$. With comparable sizes of aptamers and EDL, we expect an efficient change in the EDL composition upon binding of aptamers, which is evident from the strong threshold shifts in the transfer characteristics. ${ }^{27}$ To avoid nonspecific binding of analyte PSA, a molecular blocking layer of ethanolamine (EA) was used. This step was applied to the whole chip surface and should block the nonfunctionalized surface groups of the aptamer-modified regions as well as the nonmodified surfaces next to the microspots when the microspotting procedure was used in some experiments (for details, please refer to the Supporting Information S3). The binding of EA to the Si NW-ISFET surfaces induced a decrease in $V_{\text {th }}$ of several $100 \mathrm{mV}$ (Figure 2a). After this blocking step, the aptamer sensor platform was ready for the detection of PSA.

A PB solution containing $30 \mathrm{nM}$ enzymatically active PSA from human seminal fluid was applied to the sensor platform and allowed to bind to the sensor surfaces. The resulting transfer characteristic of a typical Si NW-ISFET is shown in Figure 2a (blue curve). It is known from conformational studies that DNA aptamers form adaptable secondary and tertiary structures to bind with their target analytes. ${ }^{48}$ These conformational binding mechanisms are responsible for a sometimes even higher specificity and affinity of aptamer receptors compared to their antibody counterparts. ${ }^{42}$ Some portions of the negatively charged phosphate backbones of the aptamers are shielded during this conformational adaptation upon binding with PSA and therefore a reduction in total density of negative surface charges on the solid-liquid interface is possible. ${ }^{43}$ It was also earlier reported that the isoelectric point of PSA can vary in a wide range from $\mathrm{pH} 6.4$ to 8 because of the variable degree of its glycosylation. ${ }^{38,57,58}$

We assume that the $V_{\text {th }}$ increase upon binding of PSA to the DNA aptamers on the Si NW surfaces is caused by a positive charge addition onto the surface. Therefore, for our experiments, the PI of this particular PSA in its glycosylation state should be higher than 7.4. The aptamer-PSA binding causes an increase in $V_{\text {th }}$ of the Si NW-ISFETs.

To confirm the specificity of the sensor signal, we applied the same $30 \mathrm{nM}$ PSA in PB solution to Si NW-ISFETs without aptamer functionalization as controls. The response of a control sensor is shown in Figure $2 \mathrm{~b}$. As can be seen from the blue and green curves of Figure 2a,b, respectively, the sensor response was highly specific. However, individual responses were underlying statistical variations. With the 32-channel Si NW-ISFET sensor chips (arranged in 8 sets of 4 Si NWs each), we were able to statistically evaluate the sensor platform. In Figure $2 c$, it is shown that the platform could also be utilized with on-chip control channels. A typical experiment is shown, where 27 out of 32 channels were electrically functioning. 

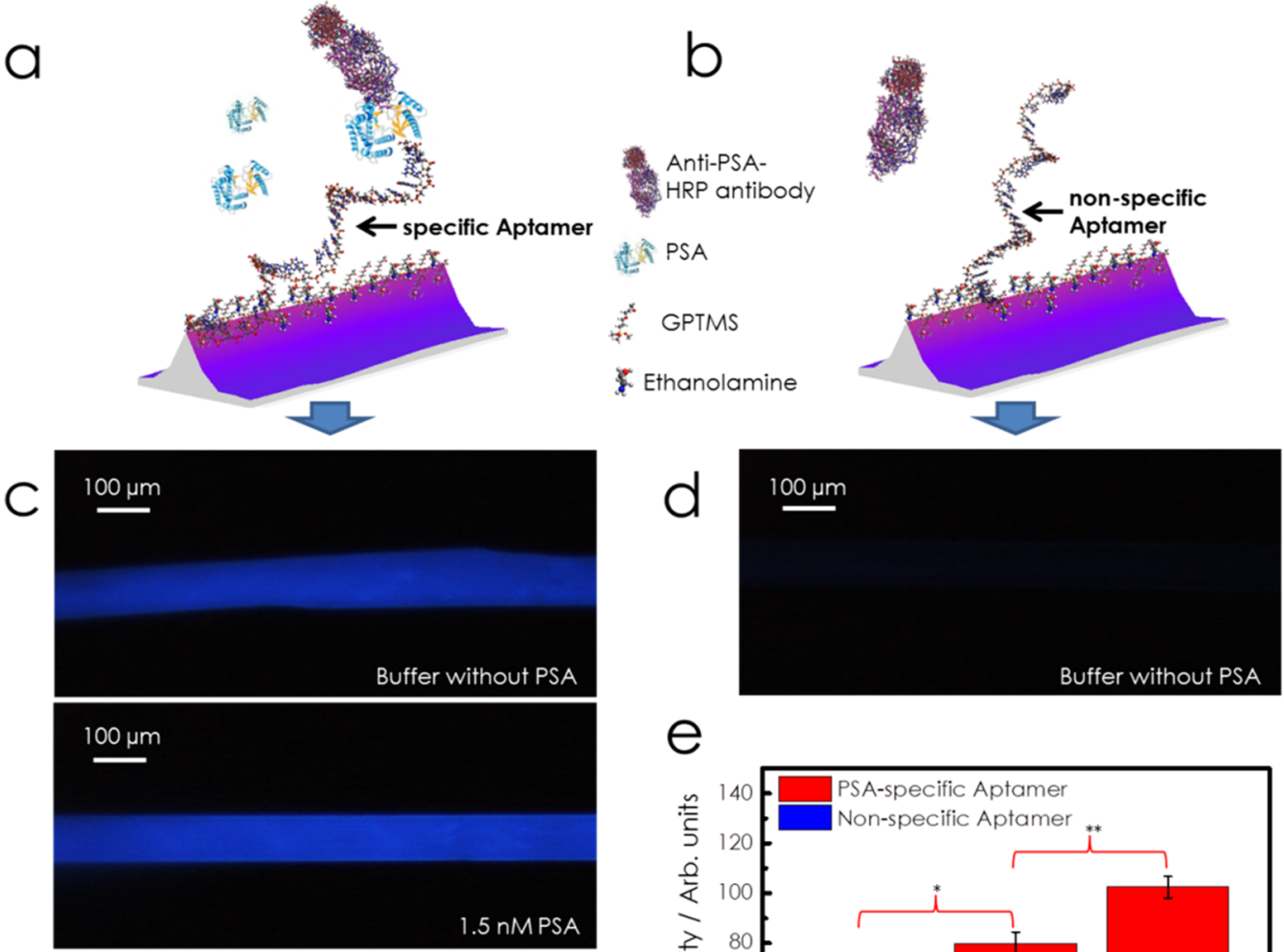

e
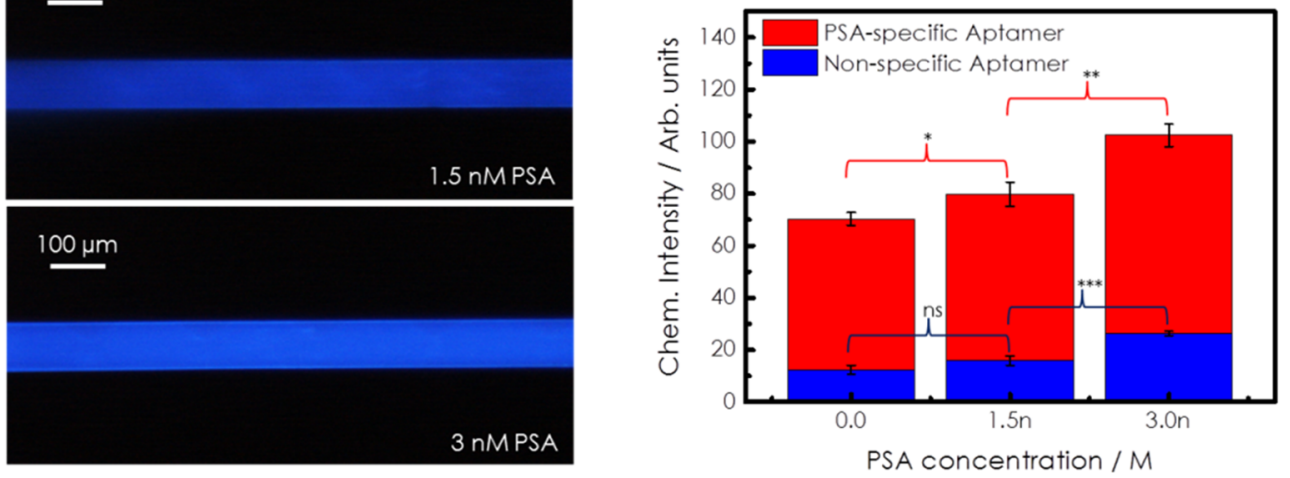

Figure 3. Testing the performance of the biofunctional layers on the Si NW-ISFET surfaces using a chemiluminescence assay: schematic illustrations showing the build-up of the biofunctional layers for the chemiluminescence assay (a, b). Microscopic images showing the chemiluminescence intensities for the detection of analyte PSA using the specific aptamer sequence. Also, in the case of PB without PSA, the antiPSA HRP antibodies bind to the specific aptamers. Nevertheless, a chemiluminescence increase with increasing PSA concentration can be seen (c). In the case of the nonspecific PSA aptamer receptors also, the anti-PSA HRP antibodies did not bind nonspecifically, showing no chemiluminescence signal (d). The histogram displays the average chemiluminescence intensity (arbitrary units) of all experiments with PSAspecific and nonspecific aptamers. A paired $t$-test shows significance only in the higher concentration level (ns for not significant, $* * *$ for $P<0.001$, $* *$ for $P<0.01$ and $*$ for $P<0.1)(\mathrm{e})$

Sensor positions from 1 to 20 were functionalized with PSAspecific aptamers, whereas 21-32 were not functionalized with the aptamer. In this experiment, the site-selective functionalization was realized with a DNA microspotter. More information about the microspotting procedure for functionalization of aptamers and the influence of the microspotting to the Si NW-ISFET performance is provided in the Supporting Information (Figure S3). This experiment also details how our platform could be utilized for the parallel detection of multiple analytes. As can be seen from the graph in Figure $2 c$, changes in $V_{\text {th }}$ values were consistent over all the sensor positions with some variations in absolute values. The changes in the $V_{\text {th }}$ values after PSA interaction were much higher for the sensor positions functionalized with the aptamer. However, because the microspotting protocol was a tedious procedure, for the statistical evaluation of all experiments described in the following, only whole-chip surfaces of Si NW-ISFETs were functionalized for better reproducibility of sensor characteristics and higher throughput in the experiments. Figure $2 \mathrm{~d}$ compares the average shifts in the $V_{\text {th }}$ value for the aptamer- functionalized and nonfunctionalized sensors. The average $V_{\text {th }}$ values of the control channels did not change (PB), whereas the aptamer functionalization of the other channels caused a shift. A paired $t$-test analysis of the sensor response confirmed nonsignificant changes (ns) for controls in comparison to aptamer-functionalized Si NWs $(* * *$ for $P<0.001)$. The blocking step with EA also caused significant changes in the sensor channels and controls, whereas the binding of $30 \mathrm{nM}$ PSA induced statistically significant changes in the sensing channels (*** for $P<0.001)$ in comparison to ns for control channels. Therefore, in later experiments, the relative PSA responses are always plotted with respect to the EA transfer characteristics.

When evaluating the individual sets of one Si NW-ISFET sensor chip (four Si NWs per each set), the variation in five different sets is shown in Figure 2e. This experiment was done with aptamer-functionalized sensors and $30 \mathrm{~nm}$ PSA in PB was applied as an analyte solution. In four out of five cases, the sensors show a significant response. The complete analysis of the response of these five $\mathrm{Si} N W$ sets is shown in Figure $2 \mathrm{f}$. 

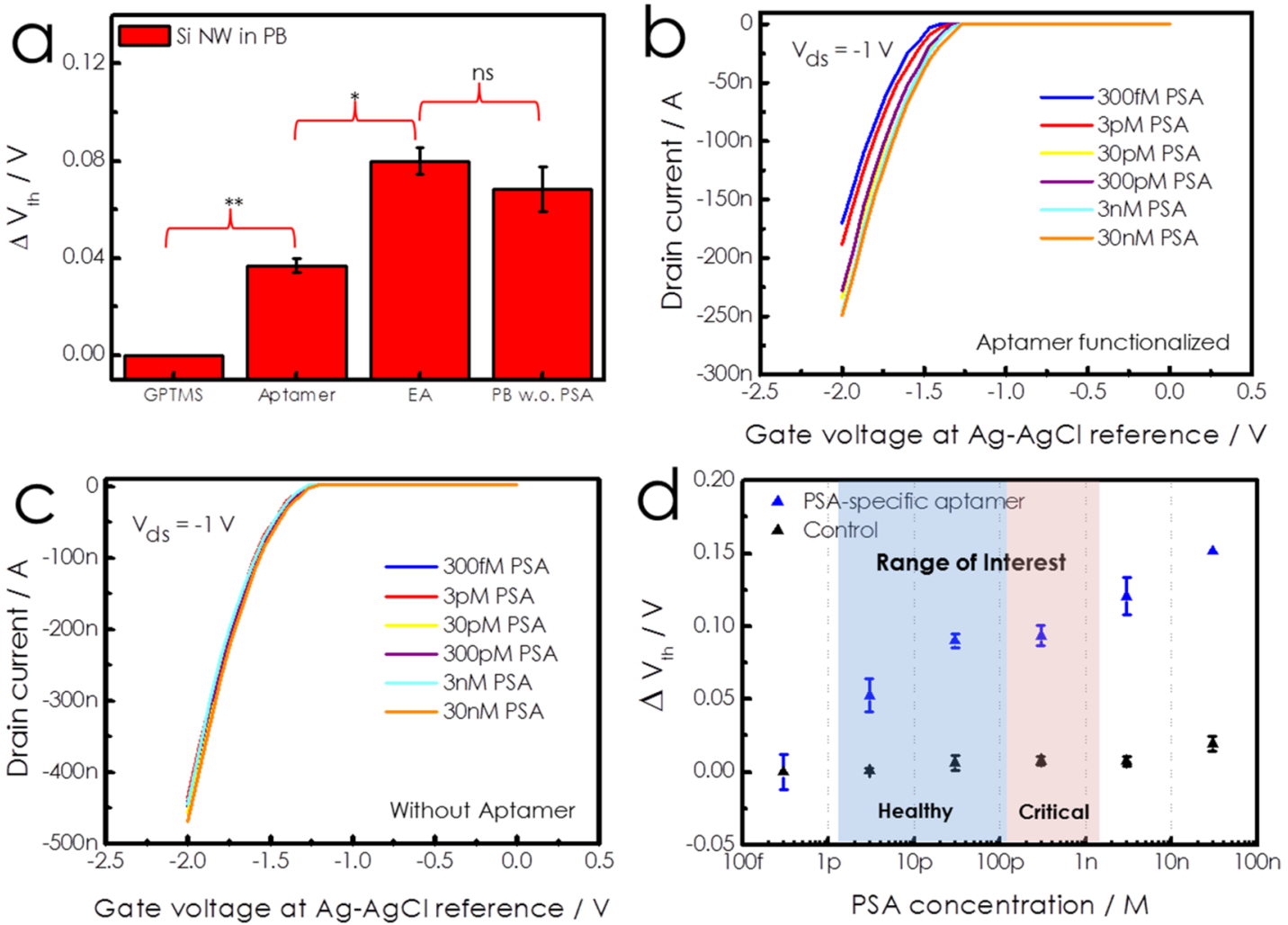

Figure 4. Label-free, fully electronic detection of PSA with Si NW-ISFETs: the histogram demonstrates how each chip was evaluated for the bioassay. Small standard deviation should be noted for multiple Si NWs from one chip. Here, a control experiment is evaluated, which shows the stability of the sensor platform. The average change in $\Delta V_{\text {th }}$ at each step of surface functionalization is displayed. The paired $t$-test results in $* *=P$ $<0.01, *=P<0.1, \mathrm{~ns}=$ nonsignificant $(n=2$ independent experiments) (a); typical field-effect response of one Si NW-ISFET functionalized with the PSA-specific aptamer and tested with PSA concentrations varying from $0.3 \mathrm{pM}$ to $30 \mathrm{nM}$ (b); typical field-effect response of a control Si NWISFET without aptamer functionalization at the same PSA concentrations confirming the PSA-specific sensor response (c). The scatter chart summarizes the absolute $V_{\text {th }}$ shifts as a function of increasing PSA concentrations for repeated experiments, where the results were baseline corrected to the smallest concentration of $0.3 \mathrm{pM}(n=2)(\mathrm{d})$. The errors bars differ because we evaluated several channels from two independent chips for the PSA concentration tests and several channels from one control chip. Data points represent average values \pm standard deviations.

Statistically significant $(* * *$ for $P<0.001$ and $* *$ for $P<0.01$ ) variations in $V_{\text {th }}$ shifts were observed at each surface modification step, as well as for the sensor response towards $30 \mathrm{nM}$ PSA. Therefore, we can conclude that the Si NWISFET platform offers stable and reproducible responses, and that it can be utilized for specific detection of PSA.

For real diagnostic applications, a PSA test should be most precise at a concentration level of around $4 \mathrm{ng} / \mathrm{mL}(0.12 \mathrm{nM})$, whereas depending on the age group and ethnic background, levels lower than $0.1 \mathrm{ng} / \mathrm{mL}(3 \mathrm{pM})$ can be measured in healthy men, but levels of $4 \mathrm{ng} / \mathrm{mL}$ and higher suggest further diagnostics in terms of biopsy. Commercially available diagnostic tests cover concentration ranges of $0-50 \mathrm{ng} / \mathrm{mL}$ $(0-1.5 \mathrm{nM})$, with limits of detection (LOD) as low as around $0.05 \mathrm{ng} / \mathrm{mL}(1.5 \mathrm{pM}) .{ }^{59}$ We defined this concentration range as the "range of interest" for our Si NW-ISFET assays. In this range, an optimum sensor for PSA detection should show highest sensitivity (i.e., sensor signal/concentration), which is the slope in the dose-response curve. At best, the relation between the sensor signal and the given concentration is linear, which is very rare for affinity-based sensor approaches. In general, it is important that the calibration curve shows a very small statistical error with good reproducibility between the data points to enable an optimum resolution of the sensor in the range of interest. From related publications in our European project PROSENSE, we know that the aptamers used in this publication show a very good binding of analyte PSA with high association constants. ${ }^{68,61}$

First, to optimize our Si NW-ISFET sensor platform toward the electrical detection of PSA with lower concentrations in the sample and to confirm the specific binding of PSA to the receptors, we utilized two optical assays with chemiluminescence and fluorescence detection, respectively. The chemiluminescence sandwich immunoassay for the detection of PSA is schematically depicted in Figure 3. Figure 3a,b shows a schematic illustration of this assay, where the Si NWs are either functionalized with PSA-specific aptamers (Figure 3a) or nonspecific aptamers (Figure $3 \mathrm{~b}$ ) of the same sequence length (32 bases) as controls. These experiments were done inside the PDMS microfluidic channels of $100 \mu \mathrm{m}$ width, which were mounted onto the Si NW-ISFET chip surfaces. By this procedure, the channel can be compared with the PDMSprotected surface as the background. The analyte PSA solution was flown through the microfluidic channels and allowed to bind to the aptamer surfaces. After this, PB solution with antiPSA horseradish peroxidase (HRP) antibodies was flown through the microfluidic channels and allowed to bind to the immobilized PSA. The substrate luminol (5-amino-2,3dihydrophthalazine-1,4-dione) was excited by oxidation to form an intermediate state using $\mathrm{H}_{2} \mathrm{O}_{2}$. The luminol in the excited intermediate state was then allowed to react with the anti-PSA HRP antibodies bound on the Si NW surfaces. The 
reaction of luminol with the HRP enzyme returned it to the ground state with an emission of a photon. This photon emission then indirectly confirmed the presence of PSA on the Si NW surfaces.

In this chemiluminescence assay, the intensity of the optical signal is directly related to the amount of anti-PSA HRP antibodies bound to PSA. It is therefore indirectly related to the concentration of PSA in the analyte solution, when a nonspecific binding of the antibodies to the surface can be excluded. ${ }^{62}$ Details of the surface modification and other procedures used in the chemiluminescence assay are provided in the Materials and Methods section. Figure $3 c$ shows the microscopic images of the chemiluminescence generated inside a microfluidic channel on the surface of a Si NW-ISFET chip upon the interaction of luminol with anti-PSA HRP antibodies. In general, this optical method is not sensitive enough for very low concentrations of PSA. The PSA concentrations of $1.5 \mathrm{nM}$ $(50 \mathrm{ng} / \mathrm{mL})$ and $3 \mathrm{nM}(100 \mathrm{ng} / \mathrm{mL})$ were tested, and significant increase in chemiluminescence intensity can only be seen with a higher concentration of PSA (Figure 3c; bottom images).

To ascertain the specificity of the optical signals, two control experiments were performed. First, the chemiluminescence intensity for the interaction between anti-PSA HRP antibodies and PSA-specific aptamers on the Si NW-ISFET surfaces was measured, such as shown in Figure $3 c$ (top image), where the PB did not contain PSA before flowing the anti-PSA HRP antibody solution through the channel. It can be seen that the anti-PSA HRP antibodies also binded unspecifically to the PSA-specific aptamers, leading to a background signal. In contrast, the nonspecific aptamer sequence did not bind the anti-PSA HRP antibodies (Figure 3d). This suggests a small cross-reactivity between anti-PSA HRP antibodies and PSAspecific aptamers, which would need to be avoided by a blocking step. For further optimization of the assay, this blocking step is a crucial element that needs more attention. However, other researchers in the field optimized such DNA aptamer assays on glass slides, ${ }^{63,64}$ and we could apply and adapt the same principles to our $\mathrm{SiO}_{2}$ sensors surfaces. The statistical evaluation of all chemiluminescence experiments that showed variation in chemiluminescence intensity as a function of PSA concentration is presented in the histogram plot of Figure 3e. Significance was confirmed for the interactions of the PSA-specific aptamers with PSA in comparison to nonspecific interactions at lower concentrations (ns for not significant, *** for $P<0.001$, ** for $P<0.01$, and * for $P<$ $0.1)$.

These results confirm that the surface chemistry on the $\mathrm{Si}$ NW-ISFET surfaces is robust, and that the aptamer receptor layer specifically captures PSA from the analyte solution. The second optical method based on direct fluorescence detection with anti-PSA fluorescein isothiocynate antibodies was less sensitive. Fluorescence assay results with $30 \mathrm{nM}$ PSA in PB are provided in the Supporting Information S4.

After the confirmation of a PSA-specific binding in the chemiluminescence assays, the Si NW-ISFET sensor platform was evaluated for the electronic detection of the ultralow concentrations of PSA. Concentrations of $0.3 \mathrm{pM}, 3 \mathrm{pM}, 30$ $\mathrm{pM}, 300 \mathrm{pM}, 3 \mathrm{nM}$, and $30 \mathrm{nM}(1 \mathrm{pg} / \mathrm{mL}$ to $1 \mu \mathrm{g} / \mathrm{mL})$ were tested. This concentration range widely covers the clinically relevant concentrations of PSA as prostate cancer biomarker in men. The sensor responses of the $\mathrm{Si}$ NW-ISFETs are summarized in Figure 4. First, we tested the stability of the platform by control measurements with $\mathrm{PB}$ solution without PSA while comparing the threshold voltage changes $\left(\Delta V_{\text {th }}\right)$ at all surface modification steps (Figure 4a). The sensors demonstrated identical characteristics for all steps with low standard deviations and statistically meaningful (paired $t$-test) shifts in $V_{\text {th }}$ (ns for nonsignificant, ** for $P<0.01$ and $*$ for $P$ $<0.1$ ). As shown in Figure $4 \mathrm{a}$, the responses were nonsignificant for the interaction of PB buffer without PSA analyte molecules. Therefore, we can exclude the eventual washout effects of the biorecognition layer. It should also be noted that the standard deviations increased from step-to-step during surface modification. From this stepwise increase, we can conclude that with each surface functionalization step, the Si NW-ISFET chip surfaces get less uniform, resulting in higher variations in the signals from different locations of the chip surface. For further optimization of the PSA assay, this increasing nonuniformity should be optimized as well.

The performance of a Si NW-ISFET with different concentrations of PSA is shown in Figure $4 \mathrm{~b}$. It can be seen that the transfer characteristics shifted toward decreasing $V_{\text {th }}$ values with increasing PSA concentration. A control Si NWISFET modified without the PSA-specific aptamers is shown in Figure $4 \mathrm{c}$. As can be seen, the transfer characteristics did not show any significant shift in $V_{\text {th }}$. Figure $4 \mathrm{~d}$ summarizes the dose-response curves of all the experiments with the Si NWISFET platform, where relative threshold voltage changes ( $\Delta V_{\text {th }}$-compared to the values after EA blocking) are plotted on the $y$-axis against the PSA concentrations ( $x$-axis) from 0.3 $\mathrm{pM}$ to $30 \mathrm{nM}$. To compare different Si NW-ISFET chips, the shifts were baseline corrected $\left(\Delta V_{\text {th }}=0 \mathrm{~V}\right)$ to the lowest PSA concentration tested. A clear and significant increase in sensor signal is already visible at a PSA concentration as low as $3 \mathrm{pM}$. Control signals remained stable until a slight increase in the highest concentration of $30 \mathrm{nM}$, which we attributed to unspecific binding of PSA to the control surfaces. In Figure 4d, the range of interest is indicated, and it can be seen that our highly sensitive $\mathrm{Si}$ NW-ISFET platform already detected significant responses in a low PSA concentration regime. It is to be noted that the values for the detection channels display several sensors from two 32-channel Si NW-ISFET chips, whereas the control displays channels from one control chip (average $\Delta V_{\text {th }} \pm$ standard deviation). Therefore, the error bars for the PSA detection are a bit larger. Unfortunately, our sensor platform yet does not show the necessary resolution in the critical concentration range of about $4 \mathrm{ng} / \mathrm{mL}(120 \mathrm{pM})$, which would be needed for critical diagnostic decisions toward eventual biopsies. However, the sensor response is already strong and statistically significant at much lower concentration levels, which was also reported in related works using the same aptamer sequences. ${ }^{60,61}$ This would eventually also allow dilution and conditioning of clinical samples with conditioning buffer before PSA tests, which is advantageous to stabilize side parameters such as ionic strength and $\mathrm{pH}$ value of the test solution. In addition, also for PSA concentrations beyond this concentration level, a further signal increase can be seen (Figure $4 \mathrm{~d}$; concentrations larger than $3 \mathrm{nM}$ ), which we attribute to unspecific binding to the PSA-specific aptamer surfaces (consistent with also the signal increase in the controls).

Similarly, ultrasensitive detection of PSA with other silicon nanowire platforms was earlier reported in literature. In Table 1 , we compare the performance of our top-down fabricated aptamer Si NW-ISFET platform with contemporary studies for 
Table 1. Performances of Other Silicon Nanowire ISFET Sensor Platforms for Label-Free Detection of PSA Described in the Scientific Literature

\begin{tabular}{lcc}
\multicolumn{1}{c}{ sensor platform } & \multicolumn{1}{c}{ performance } & references \\
trimmed Si NW-ISFETs & $50-500 \mathrm{pg} / \mathrm{mL}$ & 65 \\
$\begin{array}{l}\text { Si NW-ISFETs functionalized with } \\
\text { gold nanoparticles }\end{array}$ & $23 \mathrm{fg} / \mathrm{mL}$ to $500 \mathrm{ng} / \mathrm{mL}$ & 17 \\
$\begin{array}{l}\text { Si NW-ISFET arrays } \\
\text { complementary metal oxide }\end{array}$ & $1 \mathrm{pg} / \mathrm{mL}$ to $1 \mu \mathrm{g} / \mathrm{mL}$ & $\begin{array}{c}\text { current } \\
\text { study }\end{array}$ \\
$\begin{array}{l}\text { semiconductor compatible Si } \\
\text { NW arrays }\end{array}$ & $1 \mathrm{fg} / \mathrm{mL}$ to $1 \mathrm{ng} / \mathrm{mL}$ & 37 \\
polycrystalline Si NW-ISFETs & $5 \mathrm{fg} / \mathrm{mL}$ to $500 \mathrm{pg} / \mathrm{mL}$ & 36 \\
\hline
\end{tabular}

label-free detection of PSA with silicon nanowires. In our assay, we cover a wide concentration range with an LOD clearly below $50 \mathrm{pg} / \mathrm{mL}$, which would be the necessary value for diagnostic applications.

In general, the ultrasensitive detection of biomolecules with Si NW platforms originates from the high surface-to-volume ratio of the Si NWs and precise surface charge properties attenuated with the use of small receptor molecules such as aptamers. ${ }^{43,44,66}$ We also adapted our PSA bioassay protocol to microscale ISFETs of silicon. The sensitivity toward the detection of PSA was much lower in this case (see the Supporting Information, Figure S5).

In the future, the adaptation of our Si NW-ISFET platform to a specific diagnostic PSA test would be possible because the most sensitive regime was clearly below the concentration regime of the highest interest $(4 \mathrm{ng} / \mathrm{mL})$. The necessary sample dilution would even be advantageous for diagnostic assays because this procedure could be utilized to condition the analyte solution and control the main side parameters such as the ionic strength and $\mathrm{pH}$. By this procedure, the wide response window of our platform could be adapted to the concentration range of diagnostic interest.

\section{CONCLUSIONS}

A platform based on top-down fabricated Si NW-ISFET arrays was successfully deployed for the detection of PSA in the clinically relevant concentration range from $0.3 \mathrm{pM}$ to $30 \mathrm{nM}$. We utilized highly specific aptamers to capture PSA from diluted phosphate buffer solutions. Such aptamer receptor molecules have the advantage of being very small compared to antibodies, which is beneficial for field-effect detection on surfaces, where the distance of the analyte molecules to the surface is crucial. Other clear advantages of aptamer receptors are the robustness and storage time compared to many other approaches as described in literature. ${ }^{63,64}$ The protocol for the biofunctionalization of the sensors was carefully build up stepby-step and binding of the molecules to the surfaces was confirmed by AFM. In addition, the specific binding of PSA to the PSA-specific aptamer receptors was confirmed by chemiluminescence and fluorescence detection, which we performed inside the microfluidic channels mounted on top of the chip surfaces. Compared to two optical methods, the field-effect detection with Si NW-ISFETs method showed higher sensitivity with statistically significant shifts in threshold voltages. This fully electronic detection with our platform achieved a lower LOD as well. The fabrication protocol of the sensor platform was established in a top-down wafer-scale process resulting in $\mathrm{Si} \mathrm{NW}$-ISFETs with nearly identical sensor characteristics. This enabled a reliable and reproducible sensor performance, where we could utilize chip-to-chip controls. Using a microspotter, we also demonstrated the possibility of an on-chip control, which will be mandatory for the realization of future diagnostic assays. In addition, the highly reproducible sensor sites of our Si NW-ISFET platform would enable simultaneous detection of multiple analytes on one chip like in typical glass microarray bioassays with fluorescence readout. We demonstrated the experimental capability with a precise, site-selective microspotting procedure, but we did not utilize this technique to prime the different sensors of the Si NWISFET arrays with different capture molecules. For future optimization, the highly minaturized Si NW-ISFETs would eventually allow an integration into readout tools for mobile use because the power consumption of field-effect detection is in general and with highly miniaturized Si NW-ISFETs in particular very low. In our assay, however, we yet did not reach the necessary resolution in the most critical concentration range of $\sim 4 \mathrm{ng} / \mathrm{mL}$, which is typically associated with the onset of prostate cancer. Because the LOD was much lower than this concentration level, a sample dilution would be possible to condition the patients' serum stabilizing the $\mathrm{pH}$ value while reducing the ionic strength to boost the sensitivity of the detection. So far, we only tested in saline solution with slightly elevated salt concentration. For future developments toward clinical applications, selectivity in complex media needs to be tested. One major advantage of our platform is that in principle, it also allows for parallel analysis of multiple biomarkers. For this then, the surface functionalization and in particular the blocking step before the detection need to be optimized. ${ }^{63,64}$ Our Si NW-ISFET platform therefore provides a viable substitute for a high-throughput and multiplexed analysis toward other bioassays as well. ${ }^{67}$ Equipped with highdensity integration of sensor sites and parallel readout options, such sensor platforms are in future expected to generate actionable diagnostic information for PoC medical diagnostics.

\section{MATERIALS AND METHODS}

Materials. Amine-terminated, PSA-specific DNA aptamer $\left(5^{\prime}-\mathrm{NH}_{2}-\left(\mathrm{CH}_{2}\right)_{6}\right.$-TTTT TAAT TAAA GCTC GCCA TCAA ATAG CTTT-3' $\left.{ }^{\prime}\right)$, nonspecific aptamer $\left(5^{\prime}-\mathrm{H}_{3} \mathrm{~N}-\left(\mathrm{CH}_{2}\right)_{6}\right.$ AAAAATTAATTTCGAGCGGTAGTTTATCGAAA-3'), ethanolamine (EA), and GPTMS were purchased from SigmaAldrich, Germany. PSA was obtained from Merck Chemicals Ltd. (Beeston, U.K.). For the electronic assays, PB with $\mathrm{pH} 7.4$ and $10 \mathrm{mM}$ concentration was prepared from monohydrate phosphate and dinatrium hydrogen phosphate salts. Anti-PSA antibodies [5A6]-HRP (catalog no. Ab2446) and anti-PSAfluorescein isothiocyanate antibodies (catalog no. Ab178776) were purchased from Abcam, U.K. Luminol (SuperSignal West Femto Substrate Trial Kit 34094) was purchased from Thermo Scientific, Portugal. All the other reagents were of analytical grade. Metallic plug adapters for the microfluidic channel and capillary tubing (BTPE-90) were purchased from Instech Solomon (PA). One milliliter syringes were purchased from CODAN, Germany. The liquid flow in the microfluidic channel for the optical immunoassays was controlled using a NE-300 syringe pump from New Era (NY).

Fabrication of the Sensor Chips. The fabrication process of Si NW-ISFETs was reported earlier. ${ }^{27}$ In short, the sensor chips were fabricated on SOI wafers using a combination of nanoimprint lithography and photolithography processes. ${ }^{27}$ Depending on the dimensions of the $\mathrm{SiO}_{2}$ hard mask, which we used in our processes, we got different values for absolute 
threshold voltages of the Si NW-ISFET sensors (compare Figures 2 and 4). ${ }^{68}$ Nanowire structures comprise highly pdoped individual drains and a common source contact, which are etched out of the top silicon layer of the SOI wafers. The nanowire regions of typically $15 \mu \mathrm{m}$ length and 100-200 nm width are left intrinsic and depending on the thickness of the silicon $(40-60 \mathrm{~nm})$ are either fully or partly depleted, which leads to the accumulation type transistor characteristics. The etching profile results in trapezoid cross sections, ${ }^{69}$ which are covered by a thin $\mathrm{SiO}_{2}$ gate oxide of $6-8 \mathrm{~nm}$ in an almost wrapped around gate structure, which is partly still in contact with the BOX supporting the mechanic stability and giving the possibility for back gating. Typically, we fabricate devices with subthreshold swings of $100-120 \mathrm{mV} / \mathrm{dec},{ }^{68}$ which is far away from the ideal values of about $60 \mathrm{mV} / \mathrm{dec}$. Most likely, the quality of the gate oxide could be further improved by annealing in forming gas to decrease the subthreshold slope values. For biosensor applications, this subthreshold swing should show a minimum hysteresis. In addition, the channelto-channel and the chip-to-chip variations in the Si NW should be small to enable differential readout and robustness of the biosensor assays. Over the past years we opimized the fabrication process in different steps and published these protocols earlier. $^{27,68-71}$

The sensor chips with arrays of Si NW-ISFETs measured 10 $\mathrm{mm}$ in length and $7 \mathrm{~mm}$ in width. The chips were wire-bonded onto specially designed PCBs. The wire bonds and the contact lines were then covered with PDMS to protect them from damage.

Fabrication of the Microfluidic Channels. A soft lithography process for the realization of PDMS microfluidic channels was described in an earlier report. ${ }^{72}$ In short, an aluminum mask with fluidic channel designs was patterned using optical lithography. The fluidic channel structures were then transferred to a SU- 8 resist layer by patterning in another lithography process. The patterned SU-8 served as a mold for the production of microfluidic channels in the PDMS layer. The PDMS layer (with microchannel width $=100 \mu \mathrm{m}$, height $=20 \mu \mathrm{m}$, and length $=4 \mathrm{~mm}$ ) was sealed onto the sensor chip by subjecting both to an UV-ozone treatment for $5 \mathrm{~min}$ at $28-32 \mathrm{~mW} / \mathrm{cm}^{2}$ (UVO cleaner 144AX, Jelight Company Inc., CA). The surface-oxidized PDMS and Si NW-ISFET chips were then aligned and gently pressed to ensure bonding. After 1 day, the microfluidic devices were permanently sealed (as shown in Figure 1e,f) and ready to be used for the chemiluminescence assays.

Biofunctional Layer Immobilization. The surface modification process started with a surface activation step to increase the density of hydroxyl group on the Si NW surfaces. For this, sensor chips were treated with freshly prepared piranha solution $\left(\mathrm{H}_{2} \mathrm{O}_{2} / \mathrm{H}_{2} \mathrm{SO}_{4} 1: 3\right)$ for $10 \mathrm{~min}$ at $60^{\circ} \mathrm{C}$. A gas-phase silanization process was followed for rendering the $\mathrm{Si}$ NW surfaces with ultrathin GPTMS siloxane layers, which is detailed in our previous report. ${ }^{27}$ Eight hundred picoliter volume of $2 \mu \mathrm{M}$ PSA-specific aptamers in $10 \mathrm{mM}$ PB was used for functionalization of aptamers onto the Si NWs with or without using a microspotting device (see the Supporting Information S3). The chips were incubated in a humid environment (overnight incubation). After the incubation step, sensor chips were cleaned with $10 \mathrm{mM} \mathrm{PB}$ and blow-dried in $\mathrm{N}_{2}$. To block the remaining GPTMS functional sites, $1 \mathrm{M}$ EA solution was drop-casted over sensor chips for $30 \mathrm{~min}$, thoroughly rinsed with deionized water, and blow-dried in $\mathrm{N}_{2}$.
In the end, increasing concentrations of PSA ranging from 1 $\mathrm{pg} / \mathrm{mL}$ to $1 \mu \mathrm{g} / \mathrm{mL}$ were allowed to bind on the $\mathrm{Si} \mathrm{NW}$ ISFETs chips, each for $2 \mathrm{~h}$ in a humid environment (Supporting Information, Figure 5e). After each PSA concentration binding, the Si NW-ISFETs chips were rinsed with $10 \mathrm{mM} \mathrm{PB}$ and the electronic characterizations were done.

Electrical Measurements. For the experiments, where we compared the performance of the Si NW-ISFET platform with microscale Si ISFETs (see the Supporting Information S5), a 16-channel, portable amplifier system was used. ${ }^{20}$ A semiconductor parameter analyzer (Keithley 4200, Tektronix $\mathrm{GmbH}$, Germany) was used for the measurements of Si NWs in an ISFET configuration. An external $\mathrm{Ag} / \mathrm{AgCl}$ reference electrode (DRIREF-450) from World Precision Instruments $\mathrm{GmbH}$ was used as electrochemical gate contact. In the case of the p-type, microscale Si ISFETs, a drain-source voltage $V_{\mathrm{ds}}$ of $-2 \mathrm{~V}$ was applied, whereas the gate-source voltage $V_{\mathrm{gs}}$ was swept from 0 to $-2 \mathrm{~V}$. For the Si NW-ISFETs, a $V_{\mathrm{ds}}$ of $-100 \mathrm{mV}$ was used, whereas $V_{\mathrm{gs}}$ was swept from 0 to $-1 \mathrm{~V}$. In all the above cases, $V_{\text {th }}$ was calculated by implementing the transconductance $g_{\mathrm{m}}$ extrapolation method by plotting the first derivative of $I_{\mathrm{ds}}$. The $V_{\mathrm{th}}$ is given by the $V_{\mathrm{gs}}$ axis intercept of the linear extrapolation of $g_{\mathrm{m}}$.

Chemiluminescence Assays. Twenty micromolar aptamer solution $(0.5 \mu \mathrm{L} / \mathrm{min}$ for $15 \mathrm{~min})$ was flown through the microfluidic channels for covalent immobilization of the receptor layer. This step was followed by rinsing $(5 \mu \mathrm{L} / \mathrm{min}$ for 1 min with PB) and blocking of the exposed GPTMS sites using $16 \mathrm{mM}$ EA $(0.5 \mu \mathrm{L} / \mathrm{min}$ for $5 \mathrm{~min})$. The Si NWs were then rinsed with $\mathrm{PB}(5 \mu \mathrm{L} / \mathrm{min}$ for $1 \mathrm{~min})$. Thereafter, the sample solutions with different concentrations of PSA were flown into the microfluidic channels. In the last step, a solution with $100 \mu \mathrm{g} / \mathrm{mL}$ anti-PSA-HRP antibodies was flown through the channels. A flow rate of $10 \mu \mathrm{L} / \mathrm{min}$ was used for luminol to generate the chemiluminescence and during microscopic detection. In control experiments, PB solution with $20 \mu \mathrm{M}$ of a nonspecific aptamer sequence $\left(5^{\prime}-\mathrm{H}_{3} \mathrm{~N}-\left(\mathrm{CH}_{2}\right)_{6}\right.$-AAAAATTAATTTCGAGCGGTAGTTTATCGAAA-3') was used instead of the PSA-specific DNA aptamers and flown at the same rate as with the PSA-specific aptamer surfaces.

Chemiluminescence Detection. A Leica DMLM fluorescence microscope connected to a Leica DFC300FX digital camera was used for imaging the microfluidic channels and recording the images. For the optical images, an exposure time of $200 \mathrm{~ms}$ was used with $1 \times$ optical gain. The chemiluminescence signal was recorded with an exposure time of $10 \mathrm{~s}$ and $10 \times$ optical gain, all in a dark background. The acquired images were analyzed using ImageJ software (National Institute of Health). Each PSA concentration was tested by two independent experiments with respective controls and the values shown in Figure 3c,d correspond to an average of three regions of interest in each experiment after the subtraction of the background signal.

\section{ASSOCIATED CONTENT}

\section{S Supporting Information}

The Supporting Information is available free of charge on the ACS Publications website at DOI: 10.1021/acsomega.8b00990.

Three-dimensional view of the surface functionalization steps comparing electrical and optical assays, AFM 
characterization of a stepwise functionalized $\mathrm{Si}$ NW device, a more detailed description of the microspotting procedure, experimental data of the fluorescence immunoassay using anti-PSA-fluorescein isothiocyanate antibodies and an electronic experiment with microscale ISFETs as comparison to the Si NW-ISFET experiments discussed in the main manuscript (PDF)

\section{AUTHOR INFORMATION}

\section{Corresponding Author}

*E-mail: ingebrandt@iwe1.rwth-aachen.de.

\section{ORCID $\odot$}

Vivek Pachauri: 0000-0003-3275-2429

Pedro Estrela: 0000-0001-6956-1146

Sven Ingebrandt: 0000-0002-0405-2727

\section{Present Addresses}

OHarvard Medical School, Wyss Institute for Biologically Inspired Engineering at Harvard University, Boston, 02215 Massachusetts, United States (P.W.).

$\nabla_{\text {Science for Life Laboratory, Department of Biochemistry and }}$ Biophysics, Stockholm University, Tomtebodavägen 23 A, SE17165 Solna, Sweden (N.M.).

\#Institute of Materials in Electrical Engineering 1, RWTH Aachen University, Sommerfeldstr. 24, 52074 Aachen, Germany (V.P.) (X.-T.V.) (S.I.).

${ }^{\perp}$ Material Research and Technology Department, Luxembourg Institute of Science and Technology, 41 Rue Du Brill, L-4422 Belvaux, Luxembourg (D.P.).

\section{Author Contributions}

The manuscript was prepared by D.R. under the supervision of V.P. and S.I. The fabrication process for the Si NW-ISFET sensor chips was done by D.R. with contributions from Achim Müller, X.-T.V., and V.P. Experiments were done by D.R. with help of N.M., P.J., and V.P. P.E., V.C., J.P.C., and S.I. collaborated in the framework of the European Commission FP7 program in the Marie Curie Initial Training Network PROSENSE (grant no. 317420, 2012-2016, www.prosenseitn.eu). This project stimulated scientific cooperation and exchange of researchers among the three first listed institutes and universities. P.E., V.C., J.P.C., and S.I. supervised the project as PIs, coordinated the scientific exchanges and contributed to the work by combining their groups' expertize in terms of field-effect sensing, DNA aptamer assays, microfluidics, and optical bioassays. All authors discussed the presented results and contributed toward the preparation of this manuscript.

\section{Funding}

D.R., N.M., and P.J. were funded by the project PROSENSE (grant no. 317420, 2012-2016, www.prosense-itn.eu). V.P. thanks the Euroimmun AG and the Stiftung Rheinland-Pfalz für Innovation (grant no. 1082) for funding of his position.

\section{Notes}

The authors declare no competing financial interest.

This research was conducted at the University of Applied Sciences Kaiserslautern (UASK), Germany, as prime affiliation and partly at the University of Bath, U.K. and at the INESC Microsistemas e Nanotecnologias (INESC), Lisbon, Portugal, during research stays of Dipti Rani.

\section{ACKNOWLEDGMENTS}

All authors thank Ruben Soares (INESC, Lissabon, Portugal) for the help with the fluidic design and the optical assays and thank Ruben Lanche for the help with the PCB design. In addition, we thank Detlev Cassel, Achim Müller, and Rainer Lilischkis (all University of Applied Sciences Kaiserslautern, Germany) for their help in the clean-room processes and with surface characterizations.

\section{REFERENCES}

(1) Zhang, A.; Lieber, C. M. Nano-Bioelectronics. Chem. Rev. 2016, 116, 215-257.

(2) Penner, R. M. Chemical sensing with nanowires. Annu. Rev. Anal. Chem. 2012, 5, 461-485.

(3) Cherstvy, A. G. Detection of DNA hybridization by field-effect DNA-based biosensors: mechanisms of signal generation and open questions. Biosens. Bioelectron. 2013, 46, 162-170.

(4) Chartuprayoon, N.; Zhang, M.; Bosze, W.; Choa, Y. H.; Myung, N. V. One-dimensional nanostructures based bio-detection. Biosens. Bioelectron. 2015, 63, 432-443.

(5) Zhang, G. J.; Ning, Y. Silicon nanowire biosensor and its applications in disease diagnostics: a review. Anal. Chim. Acta 2012, $749,1-15$.

(6) Mu, L.; Chang, Y.; Sawtelle, S. D.; Wipf, M.; Duan, X.; Reed, M. A. Silicon Nanowire Field-Effect Transistors - A Versatile Class of Potentiometric Nanobiosensors. IEEE Access 2015, 3, 287-302.

(7) Li, B.-R.; Chen, C.-C.; Kumar, U. R.; Chen, Y.-T. Advances in nanowire transistors for biological analysis and cellular investigation. Analyst 2014, 139, 1589-1608.

(8) Wipf, M.; Stoop, R. L.; Navarra, G.; Rabbani, S.; Ernst, B.; Bedner, K.; Schönenberger, C.; Calame, M. Label-Free FimH Protein Interaction Analysis Using Silicon Nanoribbon BioFETs. ACS Sens. 2016, 1, 781-788.

(9) Kuan, D. H.; Wang, I. S.; Lin, J. R.; Yang, C. H.; Huang, C. H.; Lin, Y. H.; Lin, C. T.; Huang, N. T. A microfluidic device integrating dual CMOS polysilicon nanowire sensors for on-chip whole blood processing and simultaneous detection of multiple analytes. Lab Chip 2016, 16, 3105-3113.

(10) Krivitsky, V.; Zverzhinetsky, M.; Patolsky, F. AntigenDissociation from Antibody-Modified Nanotransistor Sensor Arrays as a Direct Biomarker Detection Method in Unprocessed Biosamples. Nano Lett. 2016, 16, 6272-6281.

(11) Zhang, G.-J.; Zhang, G.; Chua, J. H.; Chee, R.-E.; Wong, E. H.; Agarwal, A.; Buddharaju, K. D.; Singh, N.; Gao, Z.; Balasubramanian, N. DNA Sensing by Silicon Nanowire: Charge Layer Distance Dependence. Nano Lett. 2008, 8, 1066-1070.

(12) Stern, E.; Wagner, R.; Sigworth, F. J.; Breaker, R.; Fahmy, T. M.; Reed, M. A. Importance of the Debye Screening Length on Nanowire Field Effect Transistor Sensors. Nano Lett. 2007, 7, 34053409.

(13) Li, B.-R.; Hsieh, Y.-J.; Chen, Y.-X.; Chung, Y.-T.; Pan, C.-Y.; Chen, Y.-T. An Ultrasensitive Nanowire-Transistor Biosensor for Detecting Dopamine Release from Living PC12 Cells under Hypoxic Stimulation. J. Am. Chem. Soc. 2013, 135, 16034-16037.

(14) Gao, N.; Zhou, W.; Jiang, X.; Hong, G.; Fu, T. M.; Lieber, C. $\mathrm{M}$. General strategy for biodetection in high ionic strength solutions using transistor-based nanoelectronic sensors. Nano Lett. 2015, 15, 2143-2148.

(15) Pachauri, V.; Ingebrandt, S. Biologically sensitive field-effect transistors: from ISFETs to NanoFETs. Essays Biochem. 2016, 60, $81-90$.

(16) Gutiérrez-Sanz, Ó.; Andoy, N. M.; Filipiak, M. S.; Haustein, N.; Tarasov, A. Direct, Label-Free, and Rapid Transistor-Based Immunodetection in Whole Serum. ACS Sens. 2017, 2, 1278-1286.

(17) Presnova, G.; Presnov, D.; Krupenin, V.; Grigorenko, V.; Trifonov, A.; Andreeva, I.; Ignatenko, O.; Egorov, A.; Rubtsova, M. Biosensor based on a silicon nanowire field-effect transistor functionalized by gold nanoparticles for the highly sensitive 
determination of prostate specific antigen. Biosens. Bioelectron. 2017, $88,283-289$.

(18) Schasfoort, R. B. M.; Streekstra, G. J.; Bergveld, P.; Koyman, R. P. H.; Greve, J. Influence of Immunological Precipitate on DC and AC behavior of an ISFET. Sens. Actuators 1989, 18, 119-129.

(19) Kulkarni, G. S.; Zhong, Z. Detection beyond the Debye screening length in a high-frequency nanoelectronic biosensor. Nano Lett. 2012, 12, 719-723.

(20) GhoshMoulick, R.; Vu, X. T.; Gilles, S.; Mayer, D.; Offenhäusser, A.; Ingebrandt, S. Impedimetric detection of covalently attached biomolecules on field-effect transistors. Phys. Status Solidi A 2009, 206, 417-425.

(21) Huang, Y. W.; Wu, C. S.; Chuang, C. K.; Pang, S. T.; Pan, T. M.; Yang, Y. S.; Ko, F. H. Real-time and label-free detection of the prostate-specific antigen in human serum by a polycrystalline silicon nanowire field-effect transistor biosensor. Anal. Chem. 2013, 85, $7912-7918$.

(22) Tran, D. P.; Wolfrum, B.; Stockmann, R.; Pai, J. H.; Pourhassan-Moghaddam, M.; Offenhausser, A.; Thierry, B. Complementary metal oxide semiconductor compatible silicon nanowires-ona-chip: fabrication and preclinical validation for the detection of a cancer prognostic protein marker in serum. Anal. Chem. 2015, 87, $1662-1668$.

(23) Kim, A.; Ah, C. S.; Park, C. W.; Yang, J. H.; Kim, T.; Ahn, C. G.; Park, S. H.; Sung, G. Y. Direct label-free electrical immunodetection in human serum using a flow-through-apparatus approach with integrated field-effect transistors. Biosens. Bioelectron. 2010, 25, 1767-1773.

(24) Stern, E.; Vacic, A.; Rajan, N. K.; Criscione, J. M.; Park, J.; Ilic, B. R.; Mooney, D. J.; Reed, M. A.; Fahmy, T. M. Label-free biomarker detection from whole blood. Nat. Nanotechnol. 2010, 5, 138-142.

(25) Mohd Azmi, M. A.; Tehrani, Z.; Lewis, R. P.; Walker, K. A.; Jones, D. R.; Daniels, D. R.; Doak, S. H.; Guy, O. J. Highly sensitive covalently functionalised integrated silicon nanowire biosensor devices for detection of cancer risk biomarker. Biosens. Bioelectron. 2014, 52, 216-224.

(26) Schwartz, M.; Nguyen, T. C.; Vu, X. T.; Weil, M.; Wilhelm, J.; Wagner, P.; Thoelen, R.; Ingebrandt, S. DNA detection with topdown fabricated silicon nanowire transistor arrays in linear operation regime. Phys. Status Solidi A 2016, 213, 1510-1519.

(27) Rani, D.; Pachauri, V.; Mueller, A.; Vu, X. T.; Nguyen, T. C.; Ingebrandt, $\mathrm{S}$. On the Use of Scalable NanoISFET Arrays of Silicon with Highly Reproducible Sensor Performance for Biosensor Applications. ACS Omega 2016, 1, 84-92.

(28) Madu, C. O.; Lu, Y. Novel diagnostic biomarkers for prostate cancer. J. Cancer 2010, 1, 150-177.

(29) Metcalf, G. A. D.; Shibakawa, A.; Patel, H.; Sita-Lumsden, A.; Zivi, A.; Rama, N.; Bevan, C. L.; Ladame, S. Amplification-Free Detection of Circulating microRNA Biomarkers from Body Fluids Based on Fluorogenic Oligonucleotide-Templated Reaction between Engineered Peptide Nucleic Acid Probes: Application to Prostate Cancer Diagnosis. Anal. Chem. 2016, 88, 8091-8098.

(30) Koo, K. M.; Carrascosa, L. G.; Shiddiky, M. J. A.; Trau, M. Amplification-Free Detection of Gene Fusions in Prostate Cancer Urinary Samples Using mRNA-Gold Affinity Interactions. Anal. Chem. 2016, 88, 6781-6788.

(31) Makarov, D. V.; Loeb, S.; Getzenberg, R. H.; Partin, A. W. Biomarkers for Prostate Cancer. Annu. Rev. Med. 2009, 60, 139-151.

(32) Bangma, C. H.; Roemeling, S.; Schroder, F. H. Overdiagnosis and overtreatment of early detected prostate cancer. World J. Urol. 2007, 25, 3-9.

(33) Gilgunn, S.; Conroy, P. J.; Saldova, R.; Rudd, P. M.; O'Kennedy, R. J. Aberrant PSA glycosylation-a sweet predictor of prostate cancer. Nat. Rev. Urol. 2013, 10, 99-107.

(34) Makarov, D. V.; Loeb, S.; Getzenberg, R. H.; Partin, A. W. Biomarkers for prostate cancer. Annu. Rev. Med. 2009, 60, 139-151.

(35) Stern, E.; Vacic, A.; Rajan, N. K.; Criscione, J. M.; Park, J.; Ilic, B. R.; Mooney, D. J.; Reed, M. A.; Fahmy, T. M. Label-free biomarker detection from whole blood. Nat. Nanotechnol. 2010, 5, 138-142.
(36) Huang, Y.-W.; Wu, C.-S.; Chuang, C.-K.; Pang, S.-T.; Pan, T.M.; Yang, Y.-S.; Ko, F.-H. Real-Time and Label-Free Detection of the Prostate-Specific Antigen in Human Serum by a Polycrystalline Silicon Nanowire Field-Effect Transistor Biosensor. Anal. Chem. 2013, $85,7912-7918$.

(37) Lu, N.; Gao, A.; Dai, P.; Mao, H.; Zuo, X.; Fan, C.; Wang, Y.; $\mathrm{Li}$, T. Ultrasensitive Detection of Dual Cancer Biomarkers with Integrated CMOS-Compatible Nanowire Arrays. Anal. Chem. 2015, $87,11203-11208$.

(38) Gao, N.; Zhou, W.; Jiang, X.; Hong, G.; Fu, T.-M.; Lieber, C. M. General Strategy for Biodetection in High Ionic Strength Solutions Using Transistor-Based Nanoelectronic Sensors. Nano Lett. 2015, 15, 2143-2148.

(39) Kim, A.; Ah, C. S.; Park, C. W.; Yang, J.-H.; Kim, T.; Ahn, C.G.; Park, S. H.; Sung, G. Y. Direct label-free electrical immunodetection in human serum using a flow-through-apparatus approach with integrated field-effect transistors. Biosens. Bioelectron. 2010, 25, 1767-1773.

(40) Presnova, G.; Presnov, D.; Krupenin, V.; Grigorenko, V.; Trifonov, A.; Andreeva, I.; Ignatenko, O.; Egorov, A.; Rubtsova, M. Biosensor based on a silicon nanowire field-effect transistor functionalized by gold nanoparticles for the highly sensitive determination of prostate specific antigen. Biosens. Bioelectron. 2017, $88,283-289$.

(41) Hamaguchi, N.; Ellington, A.; Stanton, M. Aptamer beacons for the direct detection of proteins. Anal. Biochem. 2001, 294, 126-131.

(42) Iliuk, A. B.; Hu, L.; Tao, W. A. Aptamer in bioanalytical applications. Anal. Chem. 2011, 83, 4440-4452.

(43) Song, S.; Wang, L.; Li, J.; Fan, C.; Zhao, J. Aptamer-based biosensors. TrAC, Trends Anal. Chem. 2008, 27, 108-117.

(44) O'Sullivan, C. K. Aptasensors-the future of biosensing? Anal. Bioanal. Chem. 2002, 372, 44-48.

(45) Lung Khung, Y.; Narducci, D. Synergizing nucleic acid aptamers with 1-dimensional nanostructures as label-free field-effect transistor biosensors. Biosens. Bioelectron. 2013, 50, 278-293.

(46) Jolly, P.; Formisano, N.; Tkáč, J.; Kasák, P.; Frost, C. G.; Estrela, P. Label-free impedimetric aptasensor with antifouling surface chemistry: A prostate specific antigen case study. Sens. Actuators, B 2015, 209, 306-312.

(47) Potyrailo, R. A.; Conrad, R. C.; Ellington, A. D.; Hieftje, G. M. Adapting Selected Nucleic Acid Ligands (Aptamers) to Biosensors. Anal. Chem. 1998, 70, 3419-3425.

(48) Hermann, T.; Patel, D. J. Adaptive Recognition by Nucleic Acid Aptamers. Science 2000, 287, 820-825.

(49) Han, Y.; Offenhäusser, A.; Ingebrandt, S. Detection of DNA hybridization by a field-effect transistor with covalently attached catcher molecules. Surf. Interface Anal. 2006, 38, 176-181.

(50) Haldrup, C.; Kosaka, N.; Ochiya, T.; Borre, M.; Hoyer, S.; Orntoft, T. F.; Sorensen, K. D. Profiling of circulating microRNAs for prostate cancer biomarker discovery. Drug Delivery Transl. Res. 2014, $4,19-30$

(51) Munkley, J.; Mills, I. G.; Elliott, D. J. The role of glycans in the development and progression of prostate cancer. Nat. Rev. Urol. 2016, $13,324-333$.

(52) Koo, K. M.; Carrascosa, L. G.; Shiddiky, M. J.; Trau, M. Amplification-Free Detection of Gene Fusions in Prostate Cancer Urinary Samples Using mRNA-Gold Affinity Interactions. Anal. Chem. 2016, 88, 6781-6788.

(53) Nguyen, T. C.; Schwartz, M.; Vu, X. T.; Blinn, J.; Ingebrandt, S. Handheld readout system for field-effect transistor biosensor arrays for label-free detection of biomolecules. Phys. Status Solidi A 2015, $212,1313-1319$.

(54) Munief, W.-M.; Heib, F.; Schmitt, M.; Lu, X.; Hempel, F.; Pachauri, V.; Hempelmann, R.; Ingebrandt, S. Large Area Silane Deposition via Gas-Phase Evaporation and High-Resolution Surface Characterization of Ultrathin Siloxanes Layers 2018, unpublished (submitted).

(55) GhoshMoulick, R.; Vu, X. T.; Gilles, S.; Mayer, D.; Offenhausser, A.; Ingebrandt, S. Impedimetric detection of covalently 
attached biomolecules on field-effect transistors. Phys. Status Solidi A 2009, 206, 417-425.

(56) Lee, J.-O.; So, H.-M.; Jeon, E.-K.; Chang, H.; Won, K.; Kim, Y. H. Aptamers as molecular recognition elements for electrical nanobiosensors. Anal. Bioanal. Chem. 2008, 390, 1023-1032.

(57) Peracaula, R.; Tabares, G.; Royle, L.; Harvey, D. J.; Dwek, R. A.; Rudd, P. M.; de Llorens, R. Altered glycosylation pattern allows the distinction between prostate-specific antigen (PSA) from normal and tumor origins. Glycobiology 2003, 13, 457-470.

(58) Zhang, W. M.; Leinonen, J.; Kalkkinen, N.; Dowell, B.; Stenman, U. H. Purification and characterization of different molecular forms of prostate-specific antigen in human seminal fluid. Clin. Chem. 1995, 41, 1567-1573.

(59) Healy, D. A.; Hayes, C. J.; Leonard, P.; McKenna, L.; O'Kennedy, R. Biosensor developments: application to prostatespecific antigen detection. Trends Biotechnol. 2007, 25, 125-131.

(60) Jolly, P.; Tamboli, V.; Harniman, R. L.; Estrela, P.; Allender, C. J.; Bowen, J. L. Aptamer-MIP hybrid receptor for highly sensitive electrochemical detection of prostate specific antigen. Biosens. Bioelectron. 2016, 75, 188-195.

(61) Tzouvadaki, I.; Jolly, P.; Lu, X. L.; Ingebrandt, S.; de Micheli, G.; Estrela, P.; Carrara, S. Label-Free Ultrasensitive Memristive Aptasensor. Nano Lett. 2016, 16, 4472-4476.

(62) Jolly, P.; Damborsky, P.; Madaboosi, N.; Soares, R. R. G.; Chu, V.; Conde, J. P.; Katrlik, J.; Estrela, P. DNA aptamer-based sandwich microfluidic assays for dual quantification and multi-glycan profiling of cancer biomarkers. Biosens. Bioelectron. 2016, 79, 313-319.

(63) Krishnan, Y.; Simmel, F. C. Nucleic Acid Based Molecular Devices. Angew. Chem., Int. Ed. 2011, 50, 3124-3156.

(64) Mok, W.; Li, Y. F. Recent Progress in Nucleic Acid AptamerBased Biosensors and Bioassays. Sensors 2008, 8, 7050-7084.

(65) Kang, G. B.; Kwon, S.-M.; Kim, Y. H.; Kim, Y. T.; Sohn, Y.-S. Sensing of Prostate-Specific Antigen Using Trimmed Silicon Nanowire Field Effect Transistors. J. Nanoelectron. Optoelectron. 2011, 6, $330-333$.

(66) Li, J.; Zhang, Y.; To, S.; You, L.; Sun, Y. Effect of Nanowire Number, Diameter, and Doping Density on Nano-FET Biosensor Sensitivity. ACS Nano 2011, 5, 6661-6668.

(67) Zheng, G.; Patolsky, F.; Cui, Y.; Wang, W. U.; Lieber, C. M. Multiplexed electrical detection of cancer markers with nanowire sensor arrays. Nat. Biotechnol. 2005, 23, 1294-1301.

(68) Müller, A.; Vu, X. T.; Pachauri, V.; Francis, L. A.; Flandre, D.; Ingebrandt, S. Wafer-Scale Nanoimprint Lithography Process Towards Complementary Silicon Nanowire Field-Effect Transistors for Biosensor Applications. Phys. Status Solidi A 2018, No. 1800234.

(69) Vu, X. T.; Stockmann, R.; Wolfrum, B.; Offenhaesser, A.; Ingebrandt, S. Fabrication and application of a microfluidic-embedded silicon nanowire biosensor chip. Phys. Status Solidi A 2010, 207, 850857.

(70) Vu, X. T.; Eschermann, J. F.; Stockmann, R.; GhoshMoulick, R.; Offenhausser, A.; Ingebrandt, S. Top-down processed silicon nanowire transistor arrays for biosensing. Phys. Status Solidi A 2009, 206, 426-434.

(71) Vu, X. T.; GhoshMoulick, R; Eschermann, J. F.; Stockmann, R.; Offenhausser, A.; Ingebrandt, S. Fabrication and application of silicon nanowire transistor arrays for biomolecular detection. Sens. Actuators, B 2010, 144, 354-360.

(72) Madaboosi, N.; Soares, R. R. G.; Chu, V.; Conde, J. P. A microfluidic immunoassay platform for the detection of free prostate specific antigen: a systematic and quantitative approach. Analyst 2015, 140, 4423-4433. 